\title{
Síndrome de Burnout em trabalhadores de Enfermagem de áreas critica
}

\author{
Burnout Syndrome in critical areas Nursing workers \\ Síndrome de Burnout en áreas críticas trabajadores de Enfermería
}

Recebido: 15/03/2021 | Revisado: 21/03/2021 | Aceito: 24/03/2021 | Publicado: 31/03/2021

\author{
Andressa Gomes Melo \\ ORCID: https://orcid.org/0000-0003-2645-1937 \\ UNIMOGI, Brasil \\ Universidade Estadual de Campinas, Brasil. \\ E-mail: profandressa@unimogi.edu.br \\ Felipe Henrique de Oliveira \\ ORCID: https://orcid.org/0000-0002-3178-0244 \\ UNIMOGI, Brasil \\ E-mail: felipehenriqueoliveira@unimogi.edu.br \\ Bruna Antunha \\ ORCID: https://orcid.org/0000-0002-5646-5654 \\ UNIMOGI, Brasil \\ E-mail: brunhaantunha@unimogi.edu.br \\ Giovanna Vallim Jorgetto \\ ORCID: https://orcid.org/0000-0001-6910-4463 \\ UNIMOGI, Brasil \\ UNIFAE, Brasil \\ Universidade Federal de São Paulo, Brasil. \\ E-mail: profgiovannavallim@unimogi.edu.br
}

\begin{abstract}
Resumo
Objetivo: Conhecer a predisposição para Síndrome de Burnout na equipe de enfermagem em setores críticos em um hospital de média complexidade filantrópico do leste paulista e correlacionar tal predisposição com o perfil sociodemográfico dos mesmos. Método: Estudo de campo, exploratório, quantitativo, de amostragem probabilística intencional, cujos dados foram coletados via instrumento Maslach Burnout Inventory (MBI), em profissionais de enfermagem alocados nos serviços de UTI Neonatal e Adulto, Unidade de Cuidados Intermediários - Adulto e Neonatal (UCI e UNCI), Pronto Socorro e Centro Cirúrgico, por serem estes setores que prestam assistência de maior complexidade no hospital em questão. Resultados: Participaram deste estudo 83 participantes profissionais de enfermagem. Perfil sociodemográfico evidenciou prevalência do sexo feminino, faixa etária entre 26 a 40 anos, cargo de técnico em enfermagem e tempo de formação e função acima de cinco anos. A presença de SB na amostra em estudo apresentou baixo nível para as dimensões exaustão emocional e despersonalização e nível moderado para a realização profissional. Conclusão: A relação entre predisposição para Síndrome de Burnout na equipe de enfermagem de setores críticos onde o estudo foi realizado se fez presente.
\end{abstract}

Palavras-chave: Burnout; Enfermagem; Esgotamento profissional; Equipe de enfermagem.

\begin{abstract}
Objective: To know the predisposition for Burnout Syndrome in the nursing team in critical sectors in a hospital of medium philanthropic complexity in eastern São Paulo and to correlate this predisposition with their sociodemographic profile. Method: Field study, exploratory, quantitative, of intentional probabilistic sampling, whose data were collected via the Maslach Burnout Inventory (MBI) instrument, in nursing professionals allocated in the Neonatal and Adult ICU services, Intermediate Care Unit - Adult and Neonatal ( UCI and UNCI), Emergency Room and Surgical Center, as these sectors provide the most complex assistance in the hospital in question. Results: 83 nursing professionals participated in this study. Sociodemographic profile showed prevalence of females, age group between 26 to 40 years, position of nursing technician and time of training and function above 05 years. The presence of BS in the study sample showed a low level for the dimensions emotional exhaustion and depersonalization and a moderate level for professional achievement. Conclusion: The relationship between predispositions to Burnout Syndrome in the nursing team in critical sectors where the study was conducted was present.
\end{abstract}

Keywords: Burnout; Nursing; Professional burnout; Nursing team.

\section{Resumen}

Objetivo: Conocer la predisposición al Síndrome de Burnout en el personal de enfermería de sectores críticos de un hospital de mediana complejidad filantrópica del oriente paulista y correlacionar esta predisposición con su perfil sociodemográfico. Método: Estudio de campo, exploratorio, cuantitativo, de muestreo probabilístico intencional, cuyos datos fueron recolectados mediante el instrumento Maslach Burnout Inventory (MBI), en profesionales de 
enfermería adscritos a los servicios de UCI Neonatal y Adultos, Unidad de Cuidados Intermedios - Adultos y Neonatales (UCI y UNCI), Sala de Emergencias y Centro Quirúrgico, ya que estos sectores brindan la asistencia más compleja en el hospital en cuestión. Resultados: participaron en este estudio 83 profesionales de enfermería. El perfil sociodemográfico mostró una prevalencia de mujeres, con edades entre 26 y 40 años, cargo de técnico de enfermería y tiempo de formación y función superior a cinco años. La presencia de SB en la muestra en estudio mostró un nivel bajo para las dimensiones agotamiento emocional y despersonalización y un nivel moderado para el logro profesional. Conclusión: estuvo presente la relación entre la predisposición al Síndrome de Burnout en el equipo de enfermería en los sectores críticos donde se realizó el estudio.

Palabras clave: Burnout; Enfermería; Burnout profesional; Equipo de enfermería.

\section{Introdução}

$\mathrm{O}$ conceito mais conhecido nos dias atuais que representa o estresse laboral que atinge o profissional que exige alto grau de envolvimento interpessoal direto e intenso cobrança por resultados e desempenho denomina-se como Síndrome de Burnout (SB); conceito este descrito pelas psicólogas Maslach \& Jackson em 1981 que envolve um entendimento multidimensional da exaustão profissional (Oliveira et al., 2017).

A palavra burnout vem do inglês (burn=queimar) se esgotar, e (out=exterior) até o seu limite, assim traduzindo para "queimar por completo", sendo definido como um estresse laboral que reflete no esgotamento mental e físico do trabalhador, ou seja, acaba atingindo o entusiasmo e o interesse pelo trabalho fazendo com que o mesmo tenha um prejuízo em suas atividades, relacionamentos intrapessoal e extra pessoal (Ribeiro et al., 2019).

A SB é separada em três níveis de dimensões que se interligam umas as outras denominas como: alto nível de Exaustão Emocional (EE) que representa a baixa energia e entusiasmo, dificuldades para lidar com algumas situações que causam estresse e cansaço, alto nível de Despersonalização (D) que representam atitudes negativas como despreocupação e insensibilidade em relação com outras pessoas, fazendo com que o capacitado trate seus clientes e condiscípulo de forma inclemente, e Classificação Baixa (CB) que representa a degradação de suas aptidões, insatisfação com suas realizações e êxito sobre si mesmo no emprego conduzindo para a insatisfação e desgosto (Sobral et al., 2018; Fernandes et al., 2017).

Algumas categorias profissionais que passam por altos níveis de estresse relacionado ao trabalho têm sido alvo de estudos, a exemplo dos profissionais da saúde. Estudos realizados por Souza et al. (2018) e Silveira et al. (2018) sobre SB em equipe de Enfermagem encontraram resultados como alta prevalência nas mulheres (até por ser a Enfermagem uma profissão majoritariamente feminina), condições de trabalho insuficientes, duplas jornadas de trabalho, exposição de riscos biológicos e ergonômicos frequentes e tratados de forma negligenciada pelos dirigentes dos serviços, carga psicológica alta devido paradoxo de vida e morte e sofrimento e temor, e dos pacientes sob seus cuidados, além da baixa remuneração.

Nos setores de assistência hospitalar denominados críticos, como Unidades de terapia Intensiva, Pronto Socorro, Centro Cirúrgico e Recuperação pós-anestésica, entre outros, a rotina de trabalho é marcada pela alta complexidade da assistência prestada, o que expõe a equipe de enfermagem ao estresse prolongado e acarretam repercussões a segurança do paciente, gerando índices elevados de eventos adversos relacionados a assistência prestada, aumento da permanência do paciente no serviço de saúde hospitalar, aumento de gastos relacionados a assistência, entre outros (Dutra et al., 2018)

Desta forma, objetivou-se com este estudo conhecer a predisposição para a Síndrome de Burnout na equipe de Enfermagem dos setores críticos de um hospital de média complexidade do leste paulista, assim como verificar a predisposição da síndrome de burnout em profissionais de enfermagem de áreas critica hospitalares e, correlacionar o perfil sociodemográfico com a predisposição para a síndrome de burnout em equipe de enfermagem de áreas críticas. 


\section{Metodologia}

Trata-se de um estudo descritivo-exploratório, com amostragem probabilística intencional e abordagem quantitativa (Marconi \& Lakatos, 2005), realizado em hospital filantrópico de media complexidade do leste paulista; integrado ao Sistema Único de Saúde (SUS). Os dados foram coletados no mês de junho de 2020 de profissionais de enfermagem alocados nos serviços de UTI Neonatal e Adulto, Unidade de Cuidados Intermediários - Adulto e Neonatal (UCI e UNCI), Pronto Socorro e Centro Cirúrgico, por serem estes setores que prestam assistência de maior complexidade no hospital em questão. Estas unidades contam hoje com 106 funcionários da equipe de enfermagem, sendo: 20 técnicos e 04 enfermeiros na UTI adulto, 04 técnicos e 04 enfermeiros na UCI, 28 técnicos de enfermagem no centro cirúrgico e 02 enfermeiros, 02 técnicos de enfermagem na recuperação pós-anestésica, 10 técnicos de enfermagem e 04 enfermeiros no pronto socorro e 24 técnicos de enfermagem e 08 enfermeiros na UTI Neonatal.

Para a coleta de dados foi realizada inicialmente via caracterização sociodemográfico da amostra, com instrumento que constou das variáveis, idade, sexo, função, tempo de formação, tempo de trabalho no hospital em questão, tempo de trabalho na unidade em questão, entre outras, confeccionado pelos pesquisadores responsáveis e submetido a validação de conteúdo por três juízes com experiência na área de saúde mental e/ou docência e pesquisa. Posteriormente foi aplicado o instrumento Maslach Burnout Inventory (MBI), que identifica as variáveis dependentes da Síndrome de Burnout. O MBI foi criado por Christine Maslach, psicóloga e professora universitária na Califórnia-EUA, e validado no Brasil por Benevides-Pereira em 2001 (Benevides-Pereira, 2001). A forma de pontuação de todos os itens pesquisados adota a escala do tipo Likert, que varia de 0 a 6, sendo:

- (0) nunca; (1) uma vez ao ano ou menos; (2) uma vez ao mês ou menos; (3) algumas vezes no mês; (4) uma vez por semana; (5) algumas vezes por semana e (6) todos os dias.

O escore total foi obtido pela soma dos valores (graus) atribuídos em todos os 22 itens da escala. A soma dos escores obtidos em cada item resulta em um escore total, que varia de 0 a 56, sendo de classificação alta (26 a 54 pontos) para exaustão emocional, alta (9 a 30 pontos) para despersonalização e classificação baixa (0 a 33 pontos) para realização profissional, que irão caracterizar a Síndrome de Burnout. Cada item do MBI corresponde a uma das três dimensões da síndrome (Codo \& Vasques, 1999), sendo que para a Exaustão Emocional existem 09 itens (1, 2, 3, 6, 8, 13, 14, 16 e 20), para a Despersonalização 05 itens $(5,10,11,15$ e 22) e para a Baixa Realização Pessoal 08 itens (4, 7, 9, 12, 17, 18, 19, 21), nas questões 4, 12 e 21 houve a inversão de score para facilitar o entrevistado realizar o questionário.

Para tanto, os profissionais de enfermagem foram abordados durante os turnos de trabalho nas referidas unidades, avaliados quanto à elegibilidade, convidados a participar do estudo e posteriormente encaminhados a uma sala reservada para a coleta de dados. Os pesquisadores receberam treinamento prévio quanto à aplicação do instrumento Maslach Burnout Inventory (MBI). Foram incluídos neste estudo profissionais de enfermagem acima de 18 anos, com condições de entendimento acerca do Termo de Consentimento Livre e Esclarecido (TCLE) e sem histórico de doenças psíquicas de curso atual. Quanto a análise dos dados esta se realizou por estatística descritiva e $p$-valores. Para as associações entre as variáveis categóricas foi utilizado a do teste Qui-quadrado.

As análises foram realizadas pelo software estatístico Statistical Analysis System (SAS) 9.4, conduzidas por profissional estatístico, sendo que para a realização da análise estatística foi utilizado o nível de significância de 0,05 o qual equivale a uma confiança de $95 \%$, assim como para verificar se havia diferença entre os percentuais dos dois grupos utilizamos o teste qui-quadrado e teste Exato de Fisher.

O desenvolvimento do estudo ocorrerá em conformidade com o preconizado pela Resolução no 466/12 e 510/16 do Conselho Nacional de Saúde (CNS) e o projeto de pesquisa foi encaminhado para aprovado pelo Comitê Permanente de Ética em Pesquisa com Seres Humanos da Faculdades Mogiana - UNIMOGI, obtendo parecer favorável. 
Os participantes que aceitaram participar do estudo assinaram o Termo de Consentimento Livre e Esclarecido (TCLE), em duas vias de igual teor, sendo um deles entregue ao participante do estudo e outra que deverá ficar em poder dos pesquisadores, atendendo à legislação vigente no país. Este estudo contou com autorização institucional para coleta de dados.

\section{Resultados e Discussão}

Participaram deste estudo 83 participantes profissionais de enfermagem alocados nos serviços de UTI Neonatal e Adulto, Unidade de Cuidados Intermediários - Adulto e Neonatal (UCI e UNCI), Pronto Socorro e Centro Cirúrgico, onde houve seis negativas por licença médica, cinco por afastamento de férias e 16 não quererem participar do estudo.

A Tabela 1 demonstra as características sociodemográfico elencadas pelo estudo.

Tabela 1. Caracterização sociodemográfico dos participantes. Mogi Guaçu. 2020.

\begin{tabular}{lcc}
\hline Variáveis & Total & \% \\
\hline Sexo & 14 & 16,87 \\
Masculino & 69 & 83,13 \\
Feminino & & \\
\hline Idade (anos) & 10 & 12,05 \\
$18-25$ & 60 & 72,29 \\
$26-40$ & 13 & 15,66 \\
$>41$ & & \\
\hline Cargo & 66 & 79,52 \\
Técnico de Enfermagem & 17 & 20,48 \\
Enfermeiro & & \\
\hline Tempo de Formação (anos) & 06 & 07,23 \\
$<1$ ano & 14 & 16,87 \\
$1-5$ anos & 63 & 75,90 \\
$>5$ anos & & \\
\hline Tempo de Trabalho & 15 & 18,07 \\
$<1$ ano & 23 & 27,71 \\
$1-5$ anos & 45 & 54,21 \\
\hline 5 anos & 52 & 62,65 \\
\hline Quantidade de Emprego & 29 & 34,94 \\
Um & 02 & 02,40 \\
Dois & &
\end{tabular}

Fonte: Autores (2020).

Pela tabela acima, pode-se destacar maioria feminina, sendo 72,29\% entre 26 a 40 anos e 15,66\% possuem mais de 41 anos, 12,05\% estão entre 18 e 25 anos. 66 (79,52\%) participantes ocupam o cargo de técnico em enfermagem e 17 (20,48\%) são enfermeiros. O tempo de formação dos participantes foi para 63 (75,9\%) dos participantes acima de 05 anos, 14 (16,87\%) entre 1 a 5 anos e 06 (7,23\%) com menos de um ano de formação. Quanto ao tempo de trabalho em anos, foi mais prevalente 
em $45(54,21 \%)$ participantes que estavam na função a mais de 05 anos, seguindo de $23(27,215)$ entre 1 a 5 anos e 15 $(18,07 \%)$ em tempo inferior a 01 ano. $53(62,65 \%)$ participantes referiram possuir apenas 01 emprego, $29(34,94 \%)$ dois empregos e $02(2,4 \%)$ mais de dois empregos.

Entre os participantes do estudo, a maioria era do sexo feminino, entre a faixa etária de 26 a 40 anos, corroborando nossos dados com os estudos de Oliveira et al. (2017), Vasconcelos \& Martino (2017), Vidotti, Ribeiro, Galdino \& Martins (2018) e Lima (2018), além dos dados do COREN-SP (2020) que tem registrados 1234 mulheres inscritas na cidade de MogiGuaçu/SP, confirmando a predominância de mulheres na área da enfermagem. Para Cunha et al (2016), as mulheres escolhem a profissão pela afinidade do trabalho em cuidar, sendo que ao longo de sua história, a enfermagem era majoritariamente no passado realizada por mulheres da ordem religiosa, que faziam o trabalho por caridade. Segundo Costa \& Jun (2020) esse intervalo de idade é denominado como "formação profissional", pois significa à procura da identidade profissional e da especialização, indo também de encontro aos estudos de Simões \& Bianchi (2016) e Pires et al. (2020).

Quanto ao cargo ocupado pelos participantes a maioria são técnicos de enfermagem e nossos dados mais uma vez são corroborando com o estudo de Dutra et al. (2018), na qual 77,42\% eram técnicos de enfermagem. Já Souza et al. (2018) demonstrou em seu estudo um aumento desses profissionais para um total de $83 \%$ nessa função. A discrepância entre proporção de técnicos de enfermagem e enfermeiros encontrada em nosso estudo e corroborados na literatura por Dutra et al. (2018) e Souza et al. (2018) reside no fato do Brasil possuir um trabalho de enfermagem hierarquizado, na qual a maior demanda assistencial fica destinado aos técnicos de enfermagem, indo de encontro mais uma vez de encontro aos dados do COREN-SP (2020) que aponta para a cidade de Mogi- Guaçu/SP, 1011 técnicos para 351 enfermeiros.

No que tange os profissionais da enfermagem em nosso estudo, houve uma maior prevalência desses profissionais estarem atuando em hospitais no período maior que 05 anos, sendo que Moura et al. (2019) também encontrou o mesmo achado de tempo em seu estudo, indo na contra mão de Dutra et al. (2018) que evidenciou três anos de atuação da amostra no hospital onde o estudo foi realizado. Este tempo na função, encontrado em nossa amostra, denota estabilidade profissional e pode trazer a tona a estagnação da carreira, o que pode reduzir o crescimento profissional e causar frustação, levando a baixa realização profissional e exaustão emocional, mostrando que nosso estudo está na direção certa da literatura, pois para as dimensões exaustão emocional (47\%) e despersonalização (51\%) o nível baixo para síndrome de Burnout foi mais prevalente, enquanto a realização profissional esteve em 53\% dos participantes no nível moderado de síndrome.

A jornada de trabalho mais prevalente nesse estudo foi daqueles participantes que apresentaram apenas um vinculo empregatício. Estudos semelhantes também obtiveram a mesma prevalência, a exemplo de Magalhaes et al. (2020), embora esta não seja a realidade da enfermagem brasileira, conforme estudos de Vieira \& Chinelli (2013) que retratam que a precarização do trabalho, exigências de produtividade e eficiência, relações interpessoais hierárquicas conflituosas e condições laborais inadequadas podem impactar negativamente a saúde mental dos trabalhadores. Além disso, a perda dos vínculos de solidariedade entre os trabalhadores contribui para o desgaste físico, psíquico e emocional, resultando em situações de sofrimento e adoecimento, com repercussões diretas na qualidade dos serviços prestados. Os níveis elevados de estresse entre profissionais de saúde têm sido atribuídos às caraterísticas intrínsecas ao trabalho e à lida com o sofrimento humano, em condições de trabalho inadequadas, com jornadas prolongadas e baixa remuneração, associados ao fracasso de mecanismos compensatórios de adaptação perante situações de estresse. O baixo controle sobre os aspectos administrativos e clínicos na atenção à saúde resulta em atitudes negativas sobre o ambiente de trabalho e facilita o desenvolvimento da SB, segundo Rocha (2019).

A Escala de Maslach Burnout Inventory (MBI) é uma escala que avalia a presença e intensidade da Síndrome de Burnout em profissionais atuantes em suas profissões, principalmente as da área da saúde, e os resultados de sua aplicação neste estudo estão apresentados na Tabela 2. 
Tabela 2. Presença e intensidade da síndrome de Burnout, segundo a escala de Maslach Burnout Inventory (MBI). Mogi Guaçu. 2020.

\begin{tabular}{l|ll|ll|ll|l}
\hline Dimensão & $\begin{array}{l}\text { Nível } \\
\text { Baixo } \\
(\mathbf{n})\end{array}$ & $\%$ & $\begin{array}{l}\text { Nível } \\
\text { Moderado } \\
(\mathbf{n})\end{array}$ & $\begin{array}{l}\text { Nível } \\
\text { alto } \\
(\mathbf{n})\end{array}$ & $\boldsymbol{p}$-value \\
\hline Exaustão Emocional & 39 & 46,99 & 24 & 28,92 & 20 & 24,10 & $<0,001$ \\
\hline Despersonalização & 42 & 50,60 & 26 & 31,32 & 15 & 18,07 & $<0,001$ \\
\hline Realização Profissional & 17 & 20,48 & 44 & 53,01 & 22 & 26,50 & $<0,001$ \\
\hline
\end{tabular}

Fonte: Autores (2020).

Nota-se pela Tabela 2, a significância da associação entre nível de síndrome de burnout e as dimensões da escala pelo teste qui-quadrado a 5\% de significância, sendo que para as dimensões exaustão emocional (47\%) e despersonalização (51\%) o nível baixo para síndrome de burnout foi mais prevalente, enquanto a realização profissional esteve em 53\% dos participantes no nível moderado de síndrome.

A síndrome de Burnout é um fenômeno psicossocial emergente entre os trabalhadores de enfermagem, em resposta ao complexo ambiente de trabalho em saúde em que estão inseridos (Yao et al., 2013). A síndrome constitui-se de três dimensões: exaustão emocional, entendida como ausência de energia e sentimento de esgotamento dos recursos emocionais; despersonalização, caracterizada por insensibilidade emocional; e baixa realização profissional, uma tendência dos indivíduos de auto avaliação negativa do desempenho laboral e insatisfação com o desenvolvimento profissional (Maslach \& Jackson, 1981). Logo, caracteriza-se pela perda de significado do trabalho, desmotivação, atitudes negativas e de distanciamento em relação aos outros, o que causa prejuízos no processo de trabalho em saúde (Maslach \& Leiter, 2016; Van Bogaert et al., 2013) corroborando com nossos achados.

Nos serviços de Enfermagem a SB está atrelados a más condições, ambiente insalubre, excesso de horas trabalhadas, exposição a riscos, unidos à suscetibilidade e às condições de saúde do trabalhador (Pereira, 2015; Morales \& Murillo, 2015; Bezerra et al., 2019), situações estas não referenciadas pelos participantes de nosso estudo visto termos apenas 04 (4,81\%) profissionais com escala MBI positiva para SB.

Segundo Maslach, Jackson e Leiter (2016) a SB é de natureza psicológica, sendo caracterizada por sentimentos de exaustão emocional e distanciamento relacionado à resposta prolongada aos estressores interpessoais no trabalho, incluindo três dimensões: exaustão emocional, despersonalização e diminuição da realização profissional. A exaustão emocional representa a dimensão da tensão individual do Burnout, referindo-se a sentimentos de estar sobrecarregado e esgotado de suas forças emocionais e físicas. A despersonalização representa a dimensão do contexto interpessoal do Burnout e refere-se à resposta negativa, insensível ou excessiva a vários aspectos do trabalho. Embora com baixa prevalência de SB na amostra em questão, houve sentimento de realização pessoal reduzida, corroborado por estudos a exemplo de Koy, Yunibhand, Angsuroch e Fisher (2015) que também encontraram tais resultados.

Para Hall et al. (2016) a avaliação do Burnout entre os profissionais de enfermagem torna-se fundamental pela sua influência nos resultados para o paciente, profissional e instituição. As consequências do Burnout envolvem o desempenho profissional reduzido com alta possibilidade de impacto direto na segurança do paciente e qualidade da assistência. Para a organização, devem ser considerados os custos aumentados da assistência (risco elevado para eventos adversos, aumento do tempo de permanência, insatisfação e não fidelização da clientela) e de recursos humanos (licenças, afastamentos, absenteísmo, entre outras). Para o profissional, devem ser lembradas as possíveis complicações físicas, emocionais e sociais relacionadas ao Burnout (Adriaenssens, Gucht \& Maes, 2015). 
Vale ressaltar que atividades de cuidado implicam em tensão e responsabilidades constantes e que a vivência permanente com dor, sofrimento e morte nos ambientes hospitalares pode favorecer o aparecimento de sintomas de Burnout entre profissionais de enfermagem. Somam-se, ainda, outros aspectos laborais, como sobrecarga de trabalho relacionada à dimensionamento inadequado da equipe de enfermagem frente as demandas dos pacientes, jornadas de trabalho longas e exaustivas, ambiente da prática desfavorável ao desenvolvimento da assistência de enfermagem e a exposição a diferentes fatores de risco à própria saúde, como risco biológico, risco ergonômico, risco físico, dentre outros, assim como relatam Koy, Yunibhand, Angsuroch e Fisher (2015).

A Tabela 3 apresenta a pontuação dos escores para a escala de MBI.

Tabelas 3. Participantes que pontuaram com escores de 0 a 6 para a escala de Maslach Burnout Inventory (MBI). Mogi Guaçu. 2020 .

\begin{tabular}{|c|c|c|c|}
\hline Questão & Pergunta & Total & $\%$ \\
\hline \multirow{7}{*}{$\begin{array}{l}\text { 1-Eu me sinto emocionalmente } \\
\text { sugado pelo meu trabalho }\end{array}$} & Nunca & 09 & 10,84 \\
\hline & Uma vez ao ano ou menos & 12 & 14,46 \\
\hline & Uma vez ao mês ou menos & 13 & 15,66 \\
\hline & Algumas vezes no mês & 14 & 16,86 \\
\hline & Uma vez por semana & 07 & 08,43 \\
\hline & Algumas vezes por semana & 16 & 19,28 \\
\hline & Todos os dias & 12 & 14,45 \\
\hline \multirow{7}{*}{$\begin{array}{l}\text { 2-Eu me sinto consumido no fim } \\
\text { de um dia de trabalho }\end{array}$} & Nunca & 06 & 07,23 \\
\hline & Uma vez ao ano ou menos & 05 & 06,02 \\
\hline & Uma vez ao mês ou menos & 13 & 15,66 \\
\hline & Algumas vezes no mês & 09 & 10,84 \\
\hline & Uma vez por semana & 10 & 12,05 \\
\hline & Algumas vezes por semana & 25 & 30,12 \\
\hline & Todos os dias & 15 & 18,07 \\
\hline \multirow{7}{*}{$\begin{array}{l}\text { 3-Eu me sinto fatigado quando me } \\
\text { levanto pela manhã e tenho que } \\
\text { encarar outro dia neste emprego }\end{array}$} & Nunca & 36 & 43,37 \\
\hline & Uma vez ao ano ou menos & 09 & 10,84 \\
\hline & Uma vez ao mês ou menos & 08 & 09,64 \\
\hline & Algumas vezes no mês & 12 & 14,46 \\
\hline & Uma vez por semana & 04 & 04,82 \\
\hline & Algumas vezes por semana & 07 & 08,43 \\
\hline & Todos os dias & 07 & 08,43 \\
\hline \multirow{7}{*}{$\begin{array}{l}\text { 4-Eu não consigo compreender } \\
\text { facilmente como meus pacientes se } \\
\text { sentem a respeito das coisas }\end{array}$} & Nunca & 55 & 66,26 \\
\hline & Uma vez ao ano ou menos & 16 & 19,28 \\
\hline & Uma vez ao mês ou menos & 05 & 06,02 \\
\hline & Algumas vezes no mês & 01 & 01,20 \\
\hline & Uma vez por semana & 05 & 06,02 \\
\hline & Algumas vezes por semana & 01 & 01,20 \\
\hline & Todos os dias & $\begin{array}{ll}---- \\
\end{array}$ & ----- \\
\hline \multirow{2}{*}{$\begin{array}{l}5 \text { Eu sinto que trato alguns } \\
\text { pacientes como se eles fossem }\end{array}$} & Nunca & 77 & 92,77 \\
\hline & Uma vez ao ano ou menos & 02 & 02,40 \\
\hline
\end{tabular}


objetos

6 Trabalhar com pessoas o dia inteiro é realmente uma grande tensão para mim

7-Eu lido de forma efetiva com os problemas dos meus beneficiários

8-Eu me sinto esgotado pelo meu trabalho

9-Eu sinto que influencio de forma positiva as outras pessoas através do meu trabalho

10-Eu fiquei mais insensível em relação às pessoas desde que eu peguei esse emprego

11-Eu me preocupo que este emprego esteja me endurecendo emocionalmente

\begin{tabular}{|c|c|c|}
\hline Uma vez ao mês ou menos & 01 & 01,20 \\
\hline Algumas vezes no mês & 01 & 01,20 \\
\hline Uma vez por semana & ----- & ----- \\
\hline Algumas vezes por semana & 02 & 02,40 \\
\hline Todos os dias & ----- & ----- \\
\hline Nunca & 48 & 57,83 \\
\hline Uma vez ao ano ou menos & 13 & 15,66 \\
\hline Uma vez ao mês ou menos & 06 & 07,23 \\
\hline Algumas vezes no mês & 05 & 06,02 \\
\hline Uma vez por semana & 05 & 06,02 \\
\hline Algumas vezes por semana & 02 & 02,40 \\
\hline Todos os dias & 04 & 04,82 \\
\hline Nunca & 13 & 15,66 \\
\hline Uma vez ao ano ou menos & 06 & 07,23 \\
\hline Uma vez ao mês ou menos & 06 & 07,23 \\
\hline Algumas vezes no mês & 06 & 07,23 \\
\hline Uma vez por semana & 02 & 02,40 \\
\hline Algumas vezes por semana & 12 & 14,46 \\
\hline Todos os dias & 40 & 48,19 \\
\hline Nunca & 22 & 26,50 \\
\hline Uma vez ao ano ou menos & 14 & 16,87 \\
\hline Uma vez ao mês ou menos & 09 & 10,84 \\
\hline Algumas vezes no mês & 11 & 13,25 \\
\hline Uma vez por semana & 09 & 10,84 \\
\hline Algumas vezes por semana & 10 & 12,05 \\
\hline Todos os dias & 08 & 09,64 \\
\hline Nunca & 03 & 03,61 \\
\hline Uma vez ao ano ou menos & 02 & 02,40 \\
\hline Uma vez ao mês ou menos & 03 & 03,61 \\
\hline Algumas vezes no mês & 09 & 10,84 \\
\hline Uma vez por semana & 03 & 03,61 \\
\hline Algumas vezes por semana & 14 & 16,87 \\
\hline Todos os dias & 49 & 59,04 \\
\hline Nunca & 48 & 57,83 \\
\hline Uma vez ao ano ou menos & 08 & 09,64 \\
\hline Uma vez ao mês ou menos & 06 & 07,23 \\
\hline Algumas vezes no mês & 04 & 04,82 \\
\hline Uma vez por semana & 01 & 01,20 \\
\hline Algumas vezes por semana & 03 & 03,61 \\
\hline Todos os dias & 13 & 15,66 \\
\hline Nunca & 44 & 53,01 \\
\hline Uma vez ao ano ou menos & 20 & 24,1 \\
\hline Uma vez ao mês ou menos & 03 & 03,61 \\
\hline Algumas vezes no mês & 02 & 02,40 \\
\hline
\end{tabular}


Research, Society and Development, v. 10, n. 4, e7110413935, 2021

(CC BY 4.0) | ISSN 2525-3409 | DOI: http://dx.doi.org/10.33448/rsd-v10i4.13935

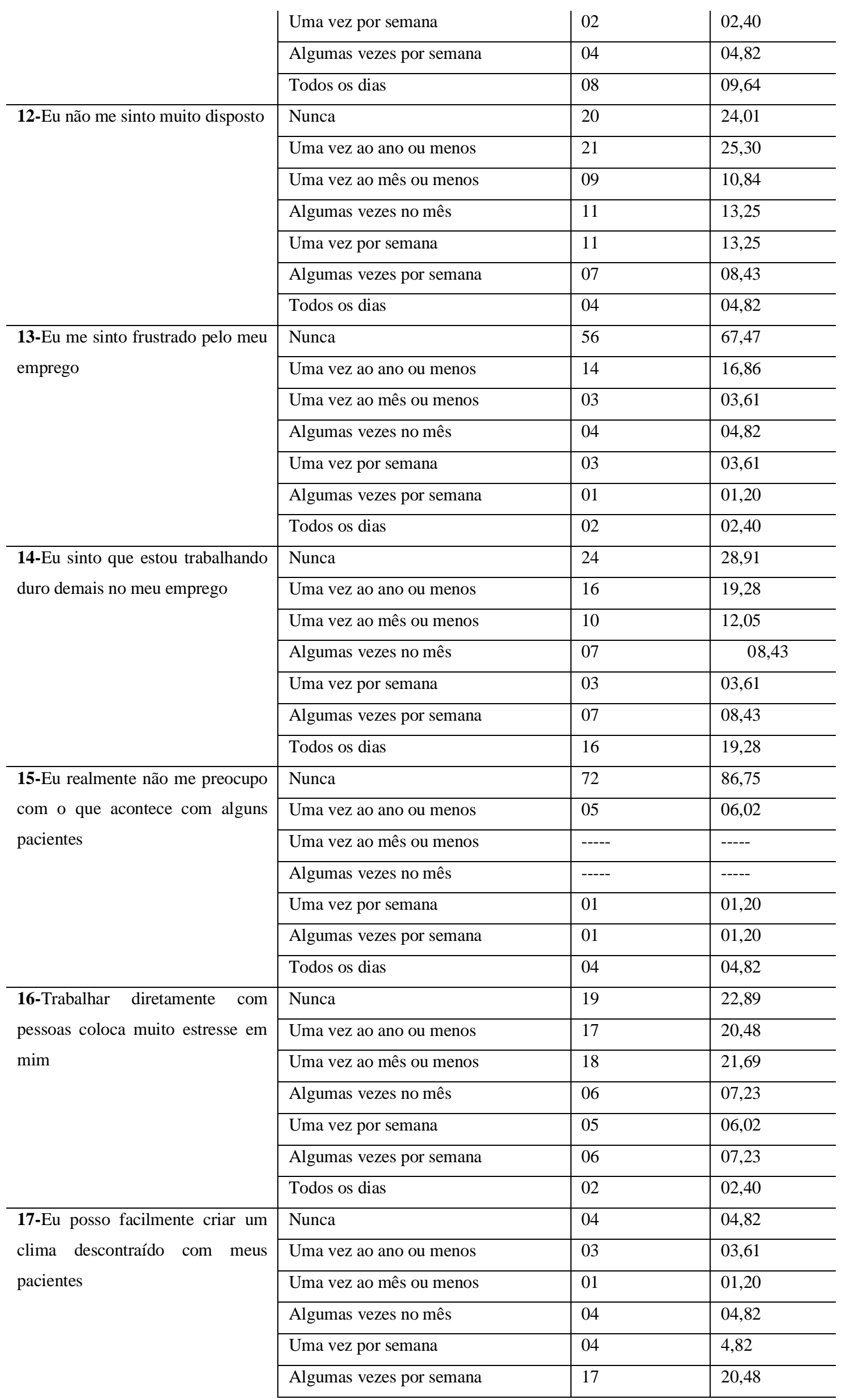




\begin{tabular}{|c|c|c|c|}
\hline & Todos os dias & 50 & 60,24 \\
\hline \multirow{7}{*}{$\begin{array}{l}\text { 18-Eu me sinto animado depois de } \\
\text { trabalhar bem próximo aos meus } \\
\text { pacientes }\end{array}$} & Nunca & 03 & 03,61 \\
\hline & Uma vez ao ano ou menos & 02 & 02,40 \\
\hline & Uma vez ao mês ou menos & 05 & 06,02 \\
\hline & Algumas vezes no mês & 03 & 03,61 \\
\hline & Uma vez por semana & 03 & 03,61 \\
\hline & Algumas vezes por semana & 23 & 27,71 \\
\hline & Todos os dias & 44 & 53,01 \\
\hline \multirow{7}{*}{$\begin{array}{l}\text { 19-Eu tenho realizado muitas } \\
\text { coisas que valem à pena neste } \\
\text { emprego }\end{array}$} & Nunca & 01 & 01,20 \\
\hline & Uma vez ao ano ou menos & 04 & 04,82 \\
\hline & Uma vez ao mês ou menos & 03 & 03,61 \\
\hline & Algumas vezes no mês & 01 & 01,20 \\
\hline & Uma vez por semana & 04 & 04,82 \\
\hline & Algumas vezes por semana & 13 & 15,66 \\
\hline & Todos os dias & 57 & 68,67 \\
\hline \multirow{7}{*}{$\begin{array}{l}\text { 20-Eu sinto como estivesse no fim } \\
\text { da linha }\end{array}$} & Nunca & 63 & 75,90 \\
\hline & Uma vez ao ano ou menos & 08 & 09,64 \\
\hline & Uma vez ao mês ou menos & 03 & 03,61 \\
\hline & Algumas vezes no mês & 04 & 04,82 \\
\hline & Uma vez por semana & ----- & ----- \\
\hline & Algumas vezes por semana & 01 & 1,20 \\
\hline & Todos os dias & 04 & 4,82 \\
\hline \multirow{7}{*}{$\begin{array}{l}\text { 21-No meu trabalho eu não lido } \\
\text { com problemas emocionais } \\
\text { tranquilamente }\end{array}$} & Nunca & 34 & 40,96 \\
\hline & Uma vez ao ano ou menos & 22 & 26,51 \\
\hline & Uma vez ao mês ou menos & 07 & 08,43 \\
\hline & Algumas vezes no mês & 05 & 06,02 \\
\hline & Uma vez por semana & 01 & 01,20 \\
\hline & Algumas vezes por semana & 05 & 06,02 \\
\hline & Todos os dias & 09 & 10,84 \\
\hline \multirow{7}{*}{$\begin{array}{l}\text { 22-Eu sinto que os pacientes me } \\
\text { culpam por alguns de seus } \\
\text { problemas }\end{array}$} & Nunca & 71 & 85,54 \\
\hline & Uma vez ao ano ou menos & 04 & 04,82 \\
\hline & Uma vez ao mês ou menos & 02 & 02,40 \\
\hline & Algumas vezes no mês & 01 & 01,20 \\
\hline & Uma vez por semana & 01 & 01,20 \\
\hline & Algumas vezes por semana & 02 & 02,40 \\
\hline & Todos os dias & 02 & 02,40 \\
\hline
\end{tabular}

Fonte: Autores (2020).

Em relação à Tabela 3, pode-se observar que as dimensões que apresentaram maiores escores, ou seja, 26-54 pontos = ALTA para exaustão emocional, 9-30 pontos = ALTA para despersonalização e de 0-33 pontos = BAIXA para realização profissional, foram: 
- Exaustão emocional referentes aos itens 1, 2, 3, 6, 8, 13, 14, 16 e 20

Baixo 0-15 $(n=24,46,99 \%$ e $<0,001)$

Médio 16 a $25(\mathrm{n}=20,28,92 \%$ e $<0,001)$

Alto 26-40 $(n=20,24,10 \%$ e $<0,001)$

- Despersonalização referentes aos itens 5, 10, 11, 15 e 22

Baixo 0-2 (n=42, 50,6\% e <0,001)

Médio 3-8 (26, 31,31\% e <0,001)

Alto 9-30 $(\mathrm{n}=\mathbf{1 5}, \mathbf{1 8 , 0 7 \%} \mathrm{e}<\mathbf{0 , 0 0 1})$

- Realização Profissional referentes aos itens 4, 7, 9, 12, 17, 18, 19, 21

Baixo 0-33 (n=17, 20,48\% e <0,001)

Médio 34-42 (n=44, 53\% e <0,001)

Alto $43-48(n=22,26,5 \%$ e $<0,001)$

Dutra et al. (2018) encontrou dados semelhantes aos nosso nas dimensões apresentadas, uma vez que a maioria dos profissionais de enfermagem apresentou níveis baixos de Burnout nas dimensões exaustão emocional (38,94\%) e despersonalização $(45,80 \%)$ e níveis moderados $(39,16 \%)$ na dimensão realização pessoal; porém ressalta-se a divergência nesta última dimensão uma vez que obtivemos baixa porcentagem.

Em revisão sistemática realizada por Adriaenssens, Gucht e Maes (2015) foi identificado que, em média, 25,9\% dos profissionais de enfermagem apresentam níveis elevados de exaustão emocional, 27,2\% referem-se a baixa realização pessoal e 34,8\% apresentaram níveis elevados de despersonalização, indo contra os nossos achados.

Montgomery, Spânu, Băban e Panagopoulou (2015) e Machado et al (2016a) trazem em seu estudo que a exaustão emocional tem sido caracterizada como central no desenvolvimento de Burnout entre profissionais de saúde, e que especificamente na enfermagem, as demandas emocionais relacionadas ao trabalho podem interferir no desempenho do profissional diante das necessidades dos pacientes, podendo ser observado comportamento de distanciamento emocional e cognitivo em relação ao trabalho como uma forma de lidar com a excessiva carga de trabalho (Dill, Erickson \& Diefendorff, 2016; Machado et al., 2016b).

Assim, a despersonalização frequentemente acompanha o sentimento de exaustão emocional. Demandas de trabalho como tempo, relacionamento interpessoal e equipes com número insuficiente de profissionais para prestar assistência podem ser considerados fatores desencadeadores de despersonalização entre os profissionais de enfermagem.

Trazem-se como limitações deste estudo as decorrentes ao delineamento do mesmo que impossibilita a relação de causa e efeito e o tamanho amostral, que podem interferir nos níveis de SB.

As contribuições se fazem a medida que a investigação do burnout em trabalhadores de enfermagem e seus fatores associados, bem como mecanismos de prevenção e enfrentamento torna-se imprescindível como instrumento de avaliação e suporte para implementação de medidas preventivas e interventivas, buscando proteger a saúde do trabalhador, e consequentemente a segurança do paciente e o sucesso organizacional.

\section{Conclusão}

A presença de SB na amostra em estudo apresentou baixo nível para as dimensões exaustão emocional e despersonalização e nível moderado para a realização profissional.

Quanto ao perfil profissional como fator predisponente ao desenvolvimento da SB destaca-se sexo feminino, idade entre 26 a 40 anos, ser técnico em enfermagem, ter tempo de formação e estar na função por tempo acima de cinco anos. 


\section{Agradecimentos}

A Faculdade Mogiana (UNIMOGI) pelo apoio na realização do presente trabalho.

\section{Referências}

Adriaenssens, J., De Gucht, V., \& Maes, S. (2015). Determinants and prevalence of burnout in emergency nurses: a systematic review of 25 years of research. International journal of nursing studies, 52(2), 649-661. https://doi.org/10.1016/j.ijnurstu.2014.11.004

Benevides-Pereira, A. M. T. (2001). Maslach Burnout Inventory e suas adaptações para o Brasil. In: $32^{a}$ Reunião Anual de Psicologia. Rio de Janeiro; 84-85. http://pepsic.bvsalud.org/scielo.php?script=sci_arttext\&pid=S0006-59432015000100006.

Bezerra, C. M. B. et al. (2019). Prevalência do estresse e síndrome de burnout em enfermeiros no trabalho hospitalar em turnos. REME Revista Mineira de Enfermagem, 23: e-1232. 9. https://doi.org/10.5935/1415-2762.20190080.

Conselho Nacional de Saúde. Resolução $\mathrm{n}^{\circ} 466$ (2012). Dispõem sobre pesquisas em seres humanos e atualiza a resolução 196. https://conselho.saude.gov.br/ultimas_noticias/2013/06_jun_14_publicada_resolucao.html.

Conselho Nacional de Saúde. Resolução nº510 (2016). Dispõem sobre ética em pesquisa aplicada as Ciências Humanas e Sociais. http://bvsms.saude.gov.br/bvs/saudelegis/cns/2016/res0510_07_04_2016.html

Coren. Conselho Regional de Enfermagem de São Paulo (2020). Numero de profissionais por categoria profissional, por município. https://portal.corensp.gov.br/enfermagem-numeros-dados.php.

Costa, D. K., \& Jun. J. Is It Me or You? (2020). A Team Approach to Mitigate Burnout in Critical Care. Critical Care Nursing Clinics. 32(3): 395-406. https://doi.org/10.1016/j.cnc.2020.05.003.

Cunha, K. L. et al. (2016). Prevalência da síndrome de burnout em profissionais da saúde que trabalham em serviço de emergência terciária com Programa de Residência pelo SUS. Revista Encontros Universitários da UFC, Fortaleza, 1(1). http://www.repositorio.ufc.br/handle/riufc/42669.

Dill, J., Erickson, R. J., \& Diefendorff, J. M. (2016). Motivation in caring labor: implications for the well-being and employment outcomes of nurses. Sociedad Science Medica: 167: 99-106. https://doi.org/10.1016/j.socscimed.2016.07.028.

Dutra, H. S. et al. (2018). Burnout entre profissionais de enfermagem em hospitais no Brasil. Revista Cuidarte, 10(1). https://revistacuidarte.udes.edu.co/index.php/cuidarte/article/view/585/1083.

Fernandes, L.S. et al. (2017). Sindrome de Burnout em profissionais de enfermagem de uma unidade de terapia intensiva. Revista Online de Pesquisa, 9(2): 551-557. https://pesquisa.bvsalud.org/portal/resource/pt/biblio-836380

Hall, L. H. et al. (2016). Healthcare staff wellbeing, burnout, and patient safety: a systematic review. PLoS ONE, 11(7): e0159015. http://dx.doi.org/10.1371/journal.pone.0159015.

Koy, V., Yunibhand, J., Angsuroch, Y., \& Fisher, M. (2015). Relationship between nursing care quality, nurse staffing, nurse job satisfaction, nurse practice environment, and burnout: literature review. Int J Res Med Sci., 3(8): 1825-31. http://dx.doi.org/10.18203/2320-6012.ijrms20150288 18203/23206012.ijrms20150288.

Lima, C. R. C. et al. (2018). Prevalência da síndrome de burnout em médicos militares de um hospital público no Rio de Janeiro. Revista Brasileira de Medicina do Trabalho, 16(3):287-96. https://doi.org/10.5327/Z1679443520180297.

Machado, M. H. et al. (2016a). Características gerais da enfermagem: o perfil sociodemográfico. Enfermagem em Foco Brasília, 7(ESP): 9-14. https://doi.org/10.21675/2357-707X.2016.v7.nESP.686 .

Machado, M. H. et al. (2016b). Mercado de trabalho da enfermagem: aspectos gerais. Enfermagem em Foco Brasília, 7(ESP): 35-62. https://doi.org/10.21675/2357-707X.2016.v7.nESP.691.

Magalhães, B. C. et al. (2020). Síndrome de burnout em uma unidade hospitalar: Percepções da equipe de enfermagem. Revista online de pesquisa: cuidado é fundamental, 12: 1004-1010. http://seer.unirio.br/index.php/cuidadofundamental/article/viewFile/7200/pdf_1.

Marconi, M. A.; \& Lakatos, E. M. (2005). Fundamentos de metodologia científica (6 ${ }^{\text {a }}$ ed), Atlas.

Maslach, C., \& Leiter, M.P. (2016). Understanding the Burnout experience: recent research and its implications for psychiatry. Wld Psychiatry. 15(2):103-11. http://dx.doi.org/10.1002/wps.20311.

Maslach, C., Schaufeli, W. B., \& Leiter, M. P. (2001). Esgotamento do trabalho. Annual Revista of Psychologic, 52: 397-422. https://doi.org/10.1146/annurev.psych.52.1.397.

Maslach, C., \& Jackson, S. (1981). The measurement of experienced Burnout. Jounal of Occupation Behavariol. 2:99-113. http://dx.doi.org/10.1002/job.4030020205. 
Montgomery, A., Spânu, F., Băban, A., \& Panagopoulou E. (2015). Job demands, burnout, and engagement among nurses: a multi-level analysis of ORCAB data investigating the moderating effect of teamwork. Burn Res. 2(2): 71-9. https://doi.org/10.1016/j.burn.2015.06.001. Acesso em: 15/10/2020

Morales, L. S., \& Murillo L. F. H. (2015). Síndrome de Burnout. Medicina Legal Costa Rica. 32(1):119-24. www.scielo.sa.cr/scielo.php?pid=S140900152015000100014\&script=sci_arttext\&tlng=en

Moura, R. S. et al. (2019). Níveis de estresse da enfermagem nas Unidades de Terapia Intensiva. Revista de enfermagem da Universidade Federal de Permanbuco on line; 13(3): 569-577. http://seer.unirio.br/index.php/cuidadofundamental/article/viewFile/7200/pdf_1.

Oliveira, R. F. et al. (2017). Incidência da Síndrome de Burnout nos profissionais de enfermagem: uma revisão integrativa. Revista de Enfermagem do CentroOeste mineiro, 7(esp. 1383). https://pesquisa.bvsalud.org/portal/resource/pt/biblio-908339.

Pereira, A. M. T. B. (2015). Elaboração e Validação do ISB- Inventario para Avaliação da síndrome de Burnout. Boletim de Psicologia, 65(142): 059-071. http://pepsic.bvsalud.org/pdf/bolpsi/v65n142/v65n142a06.pdf.

Pires, F. C. et al. (2020). Síndrome de Burnout em profissionais de enfermagem de pronto-socorro. Rev. enferm. UFPE on line: 1-7. https://pesquisa.bvsalud.org/portal/resource/pt/biblio-1102440.

Ribeiro, E. K. C. et al.(2019). Conhecimento dos profissionais de enfermagem sobre a síndrome de burnout. Revista de Enfermagem UFPE online, 13(1): 416423. https://pesquisa.bvsalud.org/portal/resource/pt/biblio-1010200.

Rocha, L. J. (2019). Esgotamento profissional e satisfação no trabalho em trabalhadores do setor de emergência e terapia intensiva em hospital público. Revista Brasileira de Medicina do Trabalho; 17(3): 300-312. https://pesquisa.bvsalud.org/portal/resource/pt/biblio-1010200.

Silveira, A. L. P. et al. (2018). Síndrome de Burnout: consequências e implicações de uma realidade cada vez mais prevalente na vida dos profissionais de saúde. Revista Brasileira de Medicina do Trabalho. v14(3): 275-284, 2018. https://cdn.publisher.gn1.link/rbmt.org.br/pdf/v14n3a13.pdf.

Simões, J., \& Bianchi L. R. O. (2016). Prevalence of the Burnout Syndrome and sleep quality of nursing technical workers. Saúde \& Pesquisa, 9(3): 473-81. http://docs.bvsalud.org/biblioref/2017/04/832986/8.pdf.

Sobral, R. C. et al. Burnout e a organização do trabalho na Enfermagem (2018). Revista Brasileira de Medicina do Trabalho, 16(1): 44-52. https://cdn.publisher.gn1.link/rbmt.org.br/pdf/v16n1a07.pdf

Souza, V. S. et al. (2018). Qualidade de vida dos profissionais de enfermagem atuantes em setores críticos. Revista de Cuidados, 9(2): 2177-2186. https://revistacuidarte.udes.edu.co/index.php/cuidarte/article/view/506/935

Van Bogaert, P. et al. (2013). The relationship between nurse practice environment, nurse work characteristics, burnout and job outcome and quality of nursing care: a cross-sectional survey. International journal of nursing studies, 50(12), 1667-1677. https://doi.org/10.1016/j.ijnurstu.2013.05.010.

Vasconcelos, E. M., \& Martino, M. M. F. (2017). Predictors of burnout syndrome in intensive care nurses. Revista Gaúcha de Enfermagem, $38(4)$, e65354. Epub June 07, 2018. https://doi.org/10.1590/1983-1447.2017.04.65354

Vidotti, V., Ribeiro, R. P., Galdino, M. J. Q., \& Martins, J. T. (2018). Burnout Syndrome and shift work among the nursing staff. Revista Latino-Americana de Enfermagem, 26, e3022. Epub August 09, 2018. https://doi.org/10.1590/1518-8345.2550.3022

Vieira, M., \& Chinelli, F. (2013). Relação contemporânea entre trabalho, qualificação e reconhecimento: repercussões sobre os trabalhadores técnicos do SUS. Ciência e Saúde Coletiva [Internet], 18(6): 1591-600. http://dx.doi.org/10.1590/S1413-81232013000600011.

Yao, Y. et al. (2013). Investigation of risk factors of psychological acceptance and Burnout syndrome among nurses in China. International of Journal Nursing Practice, 19(5):530-8. http://dx.doi.org/10.1111/ijn.12103. 